\title{
Expansion of B4GALT7 linkeropathy phenotype to include perinatal lethal skeletal dysplasia
}

\author{
Theresa Mihalic Mosher $\mathbb{D}^{1,2,3} \cdot$ Deborah A. Zygmunt $^{4}$ - Daniel C. Koboldt ${ }^{1,2} \cdot$ Benjamin J. Kelly $^{1}$. Lisa R. Johnson ${ }^{5}$. \\ David S. McKenna ${ }^{5}$ Benjamin C. Hood ${ }^{4}$ Scott E. Hickey ${ }^{2,3} \cdot$ Peter White $\mathbb{D}^{1,2} \cdot$ Richard K. Wilson ${ }^{1,2}$. \\ Paul T. Martin ${ }^{2,4} \cdot$ Kim L. McBride (iD ${ }^{2,3,6}$
}

Received: 15 February 2019 / Revised: 24 May 2019 / Accepted: 25 June 2019 / Published online: 5 July 2019

(c) The Author(s), under exclusive licence to European Society of Human Genetics 2019

\begin{abstract}
Proteoglycans have a core polypeptide connected to glycosaminoglycans (GAGs) via a common tetrasaccharide linker region. Defects in enzymes that synthesize the linker result in a group of autosomal recessive conditions called "linkeropathies". Disease manifests with skeletal and connective tissue features, including short stature, hyperextensible skin, and joint hypermobility. We report a family with three affected pregnancies showing short limbs, cystic hygroma, and perinatal death. Two spontaneously aborted; one survived 1 day after term delivery, and had short limbs, bell-shaped thorax, 11 ribs, absent thumbs, and cleft palate. Exome sequencing of the proband and one affected fetus identified compound heterozygous missense variants, NM_007255.3: c.808C >T (p.(Arg270Cys)) and NM_007255.3: c.398A >G (p.(Gln133Arg)), in B4GALT7, a gene required for GAG linker biosynthesis. Homozygosity for p.(Arg270Cys), associated with partial loss of B4GALT7 function, causes Larsen of Reunion Island syndrome (LRS), however no previous studies have linked p.(Gln133Arg) to disease. The p.(Gln133Arg) and p.(Arg270Cys) variants were transfected into CHO pgsB-618 cells. High protein expression of p.(Gln133Arg) was found, with mislocalization, compared to p.(Arg270Cys) that had a normal Golgi-like pattern. The p.(Gln133Arg) had almost no enzyme activity and little production of heparan sulfate GAGs, while p.(Arg270Cys) only had $17 \%$ of wild-type activity. These findings expand the phenotype of B4GALT7-related linkeropathies to include lethal skeletal dysplasia due to more severe loss of function.
\end{abstract}

These authors contributed equally: Paul T. Martin, Kim L. McBride

Theresa Mihalic Mosher

theresa.mihalicmosher@nationwidechildrens.org

1 Institute for Genomic Medicine at Nationwide Children's Hospital, Columbus, OH 43205, USA

2 Department of Pediatrics, The Ohio State University, Columbus, OH 43205, USA

3 Division of Genetic and Genomic Medicine, Nationwide Children's Hospital, Columbus, OH 43205, USA

4 Center for Gene Therapy, Abigail Wexner Research Institute at Nationwide Children's Hospital, Columbus, OH 43205, USA

5 Maternal-Fetal Medicine, Miami Valley Hospital, Dayton, $\mathrm{OH}$ 45409, USA

6 Center for Cardiovascular Research, Abigail Wexner Research Institute at Nationwide Children's Hospital, Columbus, OH 43205, USA

\section{Introduction}

Linkeropathies are a group of syndromes with overlapping features of skeletal dysplasias and connective tissue disorders. Multiple systems are involved, including skeletal (short stature, radio-ulnar synostosis, and decreased bone density), connective tissue (congenital contractures and dislocations, joint laxity), dysmorphisms (broad digits, brachycephaly, small mouth, prominent eyes, and short or webbed neck), and other organ involvement, including congenital heart defects and developmental delay [1,2]. Linkeropathies are caused by defects in the synthesis of proteoglycans, a major extracellular matrix component that is crucial to proper connective tissue structure and function. Proteoglycans are composed of a core protein and glycosyaminoglycan (GAG) side chains, which are attached to the protein core via a tetrasaccharide linker region (GlcA $\beta-1,3-G a l-\beta 1,3-G a l-\beta 1,4-$ Xyl- $\beta 1,3-)$. Proteoglycans, which can contain heparan sulfate, chondroitin sulfate, or dermatan sulfate GAG side chains, are a major component of the extracellular matrix in 
many tissues [1, 3]. B4GALT7 is one of at least five genes involved in the production of the linker region, and encodes galactosyltransferase I (or UDP-Galactose:O-Xylosylprotein $\beta 1,4-\mathrm{D}$-galactosyltransferase).

Pathogenic variants in B4GALT7 have been reported to cause a skeletal dysplasia, Spondylodysplastic Ehlers-Danlos syndrome (formerly referred to as the progeroid variant of EDS), and the connective tissue disorder LRS [2, 4-6]. One variant has also been described to additionally cause ocular anomalies, including corneal clouding, cataracts, and colobomas [7]. Here we describe a family with three consecutive affected pregnancies showing features of a severe, lethal connective tissue disorder/skeletal dysplasia, two of which were found to have compound heterozygous B4GALT7 variants, further expanding the phenotype of B4GALT7-related linkeropathies.

\section{Materials and methods}

\section{Exome sequencing}

Written consent was obtained prior to enrolling subjects into a research protocol approved by the Institutional Review Board at Nationwide Children's Hospital. Exome capture was performed using Agilent Clinical Research Exome reagents according to the manufacturer's protocols. Exome libraries underwent paired-end sequencing $(2 \times 151 \mathrm{bp})$ on an Illumina HiSeq 2500 instrument. We generated $\sim 15.27 \mathrm{Gbp}$ of uniquely mapped reads per sample, yielding $\sim 168 \times$ average depth across the target regions. Reads were mapped to the GRCh37 reference sequence and secondary data analysis was performed using Churchill [8], which implements the GATK "best practices" workflow for alignment, variant discovery, and genotyping. Variants were called jointly in all samples, yielding 710,602 variants $(630,349$ SNVs and 80,253 indels) that passed minimum quality filters (QUAL > 100).

SNPeff, ANNOVAR, and custom in-house scripts were used to annotate the identified variants with gene, transcript, function class, damaging scores, and population allele frequencies. Some 32,037 variants mapped to the exons or splice regions of known protein-coding genes. After removing common variants (MAF $>0.01$ in the ExAC, ESP, or 1000 Genomes databases), we selected for further analysis all splice site, frameshift, and nonsense variants, as well as missense variants predicted to be damaged by SIFT (score $<0.05$ ), Polyphen (score $>0.453$ ), GERP (score $>2.0$ ), or CADD (Phred score $>15$ ). We identified two compound heterozygous variants, NM_007255.3: c.808C $>\mathrm{T}$ (p.(Arg270Cys)) and NM_007255.3: c.398A>G (p.(Gln133Arg)), in B4GALT7 that we evaluated further with functional assays. These variants are henceforth referred to as p.(R270C) and p.(Q133R), respectively.

\section{Site-directed mutagenesis}

A plasmid containing the open reading frame of the human B4GALT7 gene was purchased from Origene (NM_007255, Rockville, MD). Primers were designed to create the patient B4GALT7 variants. For the p.(R270C) variant, the $\mathrm{C}$ was mutated to $\mathrm{T}$ at the relative human chromosome 5 position 177035995 using primer sequences 5'- GAA GAG GGA CCA GAA GTG CAT CGC AGC TCA AAA - $3^{\prime}$ and 5'TTT TGA GCT GCG ATG CAC TTC TGG TCC CTC TTC $-3^{\prime}$. For the p.(Q133R) variant, the A was mutated to $\mathrm{G}$ at the relative human chromosome 5 position 177031527 using primer sequences $5^{\prime}$ - TCT ACG TGC TCA ACC GGG TGG ACC ACT TCA G $-3^{\prime}$ and $5^{\prime}$ - CTG AAG TGG TCC ACC CGG TTG AGC ACG TAG A -3'. Site-directed mutagenesis was performed using the QuikChange II SiteDirected Mutagenesis kit following the manufacturer's protocol (Agilent Technologies, Santa Clara, CA). Clones were sequenced to confirm the presence of the desired variant.

\section{Cell culture}

pgsB-618 cells and normal CHO-K1 cells were purchased from ATCC (CRL-2241, Manassas, VA). Cells were grown at $37{ }^{\circ} \mathrm{C}$ with $5 \% \mathrm{CO}_{2}$ in Ham's $\mathrm{F} 12$ media supplemented with $10 \%$ fetal bovine serum, 50 units $/ \mathrm{mL}$ penicillin and 50 $\mu \mathrm{g} / \mathrm{mL}$ streptomycin. Some pgsB-618 cells were transfected, using Effectene according to the manufacturer's instructions (Qiagen, Germantown, MD), with plasmids containing B4GALT7-WT (wild type, WT), B4GALT7R270C (p.(R270C)), or B4GALT7-Q133R (p.(Q133R)) transgenes that allowed for constitutive expression via a cytomegalovirus promoter. All plasmids also contained a neomycin resistance gene $\left(\right.$ neo $\left.^{\mathrm{R}}\right)$ allowing for selection of stable transfectants in G418. Forty-eight hours after transfection, G418 sulfate (Corning, Manassas, VA) was added to select for transformed cells. Once selection was complete, G418 sulfate was kept in the media at a concentration of $50 \mu \mathrm{g} / \mathrm{mL}$.

\section{Immunohistochemistry}

Cells were grown on gelatin-coated coverslips in 24-well dishes for 3 days. Cells were washed once with PBS and fixed in chilled acetone for $10 \mathrm{~min}$ at $4{ }^{\circ} \mathrm{C}$. Cells were blocked in $3 \%$ BSA for 30 min at room temperature, then incubated with anti-heparan sulfate (10E4 epitope) antibody (Amsbio, Cambridge, MA), anti-GM130 antibody (BD Transduction Laboratories, San Jose, CA), or anti-calnexin 
antibody (Abcam, Cambridge, MA), and were coincubated with anti-myc tag antibody (Abcam, Cambridge, MA), for $1 \mathrm{~h}$ at room temperature. After incubation with the appropriate fluorophore-labeled secondary antibodies, the coverslips were mounted using ProLong Diamond antifade mounting solution with DAPI (Invitrogen, Carlsbad, CA).

\section{Western blot}

Cells were harvested from 6-well dishes into lysis buffer (1\% NP-40, 0.5 mM EDTA in Tris-buffered saline, $\mathrm{pH} 7.4$ with cOmplete protease inhibitor cocktail [Sigma Aldrich, St. Louis, MO]). Protein was quantitated using the microBCA assay. A fixed amount of $40 \mu \mathrm{g}$ of protein was boiled in SDS sample buffer for $10 \mathrm{~min}$ then loaded on a Bolt 4-12\% Bis-Tris Plus gel (Invitrogen, Carlsbad, CA) and transferred to nitrocellulose. The membrane was blocked in $5 \%$ nonfat dry milk in Tris-saline $(50 \mathrm{mM}$ Tris, $150 \mathrm{mM}$ $\mathrm{NaCl}$ ) containing $0.5 \%$ Tween 20 (TBST) then probed with anti-B4GALT7 antibody (Novus Biologicals, Littleton, $\mathrm{CO}$ ), anti-myc (epitope tag) antibody (Abcam, Cambridge, MA), or anti-GAPDH antibody (Sigma Aldrich, St. Louis, MO). The quantitative western blot was performed in the same way except purified B4GALT7 protein (TP300258, Origene, Rockville, MD) was loaded in 100, 50, 25, and $12.5 \mathrm{ng}$ amounts, and $50 \mu \mathrm{g}$ of cell membrane protein lysate was compared to the standard curve. Standard curves were linear and correlation coefficients exceeded 0.9. For quantitation, anti-B4GALT7 was used as the primary antibody, and IRDye $800 \mathrm{CW}$ goat anti-rabbit IgG (Li-Cor, Lincoln, $\mathrm{NE}$ ) was used for secondary detection. Imaging was done on the Odyssey CLx Infrared Imaging System (Li-Cor, Lincoln, NE).

\section{B4GALT7 enzyme activity assay}

pgsB-618 cells stably transfected with B4GALT7-WT, B4GALT7-R270C, or B4GALT7-Q133R, untransfected pgsB-618 cells, and untransfected $\mathrm{CHO}-\mathrm{K} 1$ cells were grown in 100-mm plates to near confluence. They were rinsed three times with cold PBS and detached with a cell scraper in $1 \mathrm{~mL}$ of sucrose buffer $(0.25 \mathrm{M}$ sucrose, $20 \mathrm{mM}$ Tris- $\mathrm{HCl}$ ( $\mathrm{pH} 7.5)$, cOmplete protease inhibitor cocktail (Sigma Aldrich, St. Louis, MO)). The cells were centrifuged at $1100 \times \mathrm{g}$ at $4{ }^{\circ} \mathrm{C}$ for $3 \mathrm{~min}$ and resuspended in $100 \mu \mathrm{L}$ of sucrose solution, then frozen at $-20^{\circ} \mathrm{C}$. Cell lysate suspensions were thawed rapidly at $37^{\circ} \mathrm{C}$ in a water bath and briefly sonicated at 5-s intervals for $1 \mathrm{~min}$ to produce a crude homogenate. Protein concentrations were determined by a microBCA assay. Galactosyltransferase activity was assayed in a $50 \mu \mathrm{L}$ reaction containing $2 \mu \mathrm{L}$ of DMSO, $15 \mathrm{mM} \mathrm{MnCl}_{2}, 50 \mathrm{mM} \mathrm{KCl}, 1 \%$ Triton X-100, $100 \mathrm{mM}$ MES buffer (pH 6.0), crude cell extract ( $25 \mu \mathrm{g}$ of protein),
$2 \mathrm{mM}$ p-nitrophenyl- $\beta$-D-xylopyranoside, $0.6 \mathrm{mM}$ Uridine 5 -diphosphogalactose (UDP-Gal), and $5000 \mathrm{DPM} / \mu \mathrm{L}$ (250,000 DPM/reaction) Uridine 5 -diphospho- $\left[1-{ }^{14} \mathrm{C}\right]$ galactose (American Radiolabeled Chemicals, St. Louis, MO). After incubation at $37^{\circ} \mathrm{C}$ for $1 \mathrm{~h}$, the reactions were loaded on Waters Sep-Pak C18 classic cartridges (Thomas Scientific, Swedesboro, NJ), which had been prewashed with methanol and $10 \%$ methanol. Unincorporated substrate was eluted from the cartridges with $10 \%(\mathrm{v} / \mathrm{v})$ methanol in water $(10 \mathrm{~mL})$, after which product was eluted with methanol $(5 \mathrm{~mL})$ into Ultima Gold XR scintillation fluid (Perkin Elmer, Waltham, MA). The amount of ${ }^{14} \mathrm{C}$ present in each sample was counted on the Perkin Elmer Tri-Carb 4910TR liquid scintillation counter. Significant differences between WT and p.(R270C), and between WT and p.(Q133R) values, were determined using one-way ANOVA with a post hoc Dunnett's multiple comparisons test.

\section{Results}

\section{Clinical description}

The male proband was the result of the second pregnancy and first live born child, born to healthy, nonconsanguineous parents at full term. The proband passed away on day 1 of life due to multiple congenital anomalies. Prenatal ultrasounds had shown increased nuchal translucency and edema extending down the thorax at 10 weeks gestation, progressing to a large cystic hygroma by 12 weeks. Chorionic villus sampling (CVS) was performed and chromosomal microarray and karyotype were done with normal results. By 15 weeks, fetal long bones were measuring short and abnormal posturing of the extremities was noted. At 17 weeks, it was suspected that there were missing digits on both hands based on ultrasound examination; by 30 weeks, bilateral absence of the thumbs was noted and the thoracic to abdominal circumference suggested pulmonary hypoplasia. The child was born at 40 weeks and 2 days gestation by spontaneous vaginal delivery. Apgar scores were 1 at $1 \mathrm{~min}$ and 1 at $5 \mathrm{~min}$, and the child died within an hour of delivery. Postnatal evaluation, x-ray images, and autopsy showed cleft palate, pulmonary and renal hypoplasia, horseshoe adrenal gland, and features of a skeletal dysplasia, including bell-shaped chest, 11 pairs of ribs, short extremities, short neck, absent thumbs, and clubfeet (Fig. 1). Next-generation DNA sequencing of a panel of skeletal dysplasia genes was performed on cord blood and was negative for variants that would explain the phenotype in any of the genes tested. High density targeted array was also performed as part of this panel, and no copy number variations were detected. During the pregnancy, the parents underwent InheriGen 
Fig. 1 Postnatal $x$-ray images of proband showing small chest, bowed, short extremities, dislocated joints, and bilateral absent thumbs

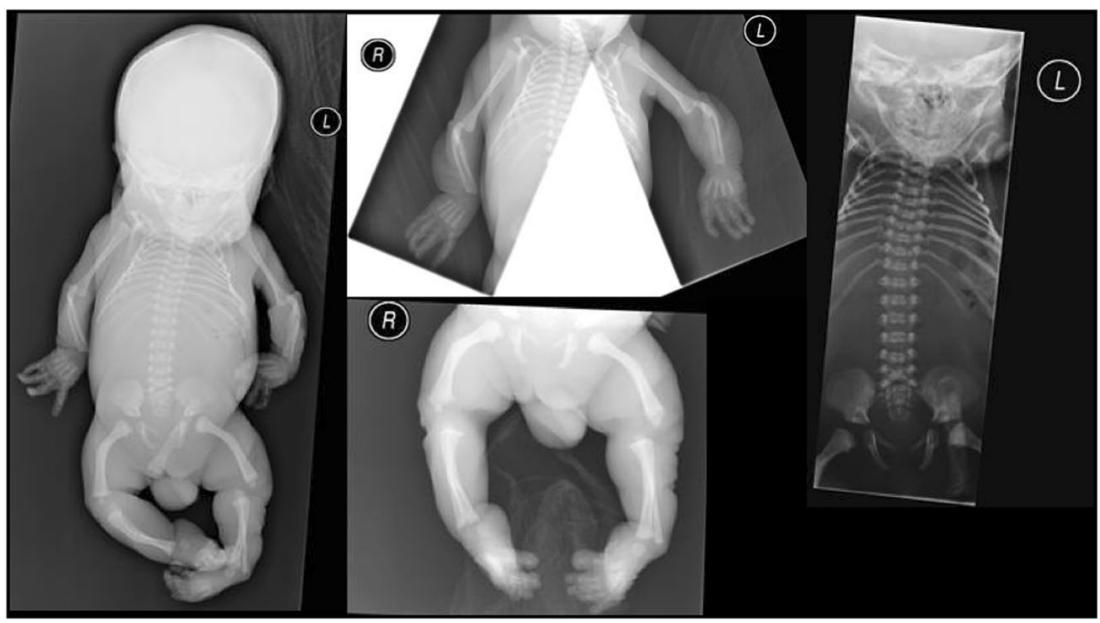

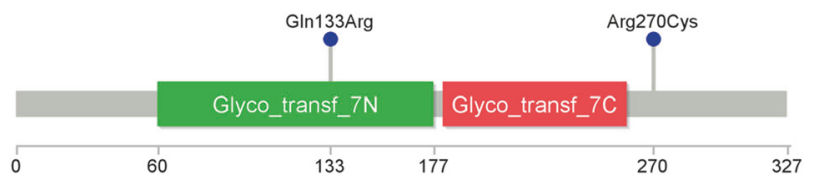

Fig. 2 Graphical view of variants detected in the proband and affected third pregnancy

carrier testing for autosomal recessive and X-linked disorders (Women's Health Laboratory, GenPath Diagnostics, Elmwood Park, NJ). There was one paternal variant identified in a gene for an autosomal recessive disorder, but nothing to explain the fetal phenotype.

Prior to the proband, the couple had a previous pregnancy (female) that resulted in a spontaneous abortion at $\sim 15$-week gestation. Cystic hygroma was seen on ultrasound at $\sim 12$-week gestation during that pregnancy. Chromosome analysis was performed on products of conception showing a 46,XX karyotype. After the proband, the couple had a subsequent pregnancy that resulted in a spontaneous abortion at $\sim 16$-week gestation. Cystic hygroma and short limbs were noted on prenatal ultrasound.

\section{Exome sequencing}

DNA from cord blood was obtained from the proband, DNA from products of conception was obtained from the third pregnancy, and DNA from peripheral blood was obtained from both parents. All four samples were used for exome sequencing.

We searched for variants consistent with recessive inheritance that were present in both the proband and affected third pregnancy, finding two compound heterozygous variants in B4GALT7. The parents were each heterozygous for one of the variants identified. The paternally inherited variant (chr5:177031527A $>\mathrm{G}$; rs1370937766) has been observed in 1 out of 120473 gnomAD individuals

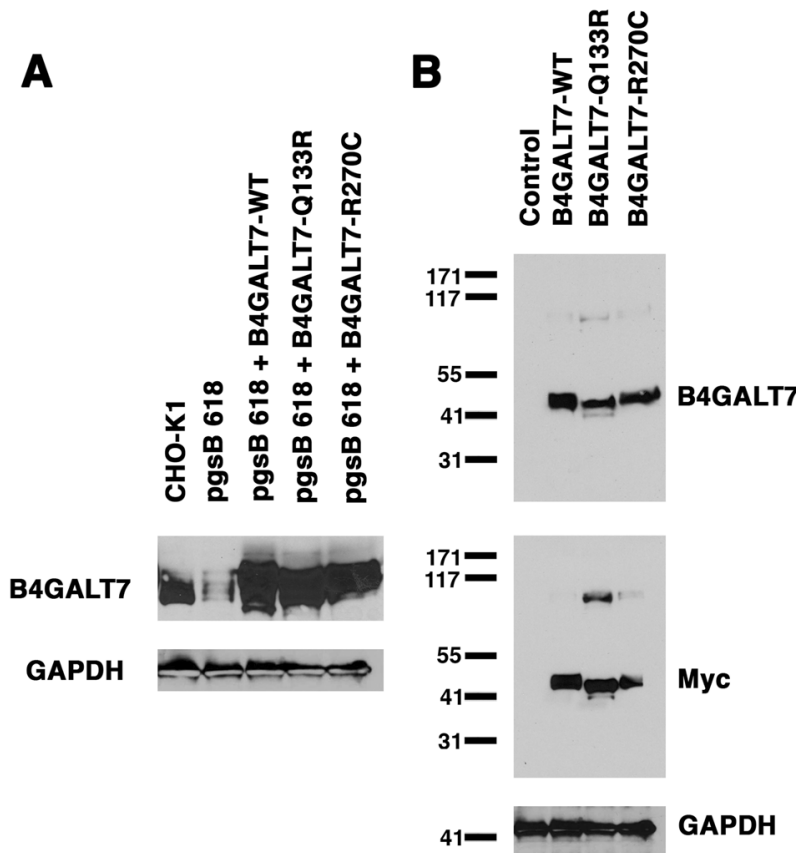

Fig. 3 Expression of B4GALT7 protein and myc epitope tag in stably transfected pgsB-618 cells. Protein lysates prepared from untransfected normal CHO cells (CHO-K1), B4GALT7-mutant pgsB-618 $\mathrm{CHO}$ cells (Control), and pgsB-618 cells stably transfected with B4GALT7-WT, B4GALT7-Q133R, or B4GALT7-R270C were blotted with anti-B4GALT7 or anti-myc antibodies. GAPDH immunoblots were used as a control for protein loading and transfer

( 1 heterozygote, $\mathrm{MAF}=0.000004)$. The variant causes a missense change, NM_007255.3: c.398A>G (p.(Q133R)), in the glycosyltransferase domain (Fig. 2) and is predicted to be damaging by $7 / 10$ algorithms in VarSome (DANN, MutationTaster, FATHMM-MKL, LRT, MutationAssessor, SIFT, and Provean). The maternally inherited variant (chr5:177035995C $>\mathrm{T}$; rs28937869) has been observed in 13 of 122110 gnomAD individuals (all heterozygotes; $\mathrm{MAF}=0.000053$ ). This is a known pathogenic variant 
previously associated with LRS. It is a missense change, NM_007255.3: c.808C $>\mathrm{T}$ (p.(R270C)), that is predicted to be damaging by $7 / 10$ algorithms in VarSome (DANN, MutationTaster, FATHMM-MKL, LRT, MutationAssessor, SIFT, and Provean). Variants were submitted to ClinVar (https://www.ncbi.nlm.nih.gov/clinvar/) under submission numbers SUB5662154 and SUB5662223.

\section{Linker synthesis assays}

In order to determine the functional effect of these variants, we began by making single point mutations in the human B4GALT7 gene that were identical to the compound heterozygous variants identified in the patients described above. In each instance, a myc epitope tag was placed at the C-terminus of the protein to allow for protein identification. Expression vectors for WT B4GALT7 (B4GALT7-WT) and for the p.(R270C) and p.(Q133R) mutants (B4GALT7$R 270 C$ and B4GALT7-Q133R, respectively) were then transfected into the $\mathrm{CHO}$ cell variant pgsB-618, which lacks endogenous B4GALT7 enzyme activity [9]. Stably transfected cells were batch selected using G418, as each expression vector also carried a neomycin resistance $\left(n e o^{R}\right)$ gene. We were able to identify a single B4GALT7 protein made for each construct using either an anti-B4GALT7 antibody or an anti-myc antibody (Fig. 3). This protein migrated at $\sim 45 \mathrm{kDa}$, slightly above the expected molecular weight of the $37 \mathrm{kDa}$ native protein. This may be in part due to the presence of the additional epitope tag or to posttranslational modifications. Western blots were stripped and reprobed for GAPDH to control for equal protein loading and transfer. We also compared total B4GALT7 protein expression in transfected pgsB-618 cells to endogenous B4GALT7 expression normal CHO-K1 cells (Fig. 3). Here, we found a 1.9, 1.6, or 1.3-fold increase in total B4GALGT7 expression in B4GALT7-WT-, B4GALT7$R 270 C$-, or B4GALT7-Q133R-transfected pgsB-618 cells relative to normal $\mathrm{CHO}-\mathrm{K} 1$ cells.

We next coimmunostained pgsB-618 cells with an antibody to heparan sulfate to identify relative amounts of heparan sulfate glycosaminoglycans made by the B4GALT7 proteins and with an antibody to myc to identify the epitope tag present on the transfected B4GALT7. Heparan sulfate staining was done with acetone fixation as previously described [10]. pgsB-618 cells expressing B4GALT7-WT showed increased heparan sulfate staining across all cells compared to untransfected cells, and anti-myc staining showed a
Fig. 4 Expression of heparan sulfate and myc-(B4GALT7) epitope tag in stably transfected pgsB-618 cells. Immunostaining with anti-heparan sulfate antibody (green) and anti-myctag antibody (red) in untransfected pgsB-618 cells and pgsB-618 cells stably transfected with B4GALT7-WT, B4GALT7-Q133R, or B4GALT7-R270C. Merge + DAPI shows a three-color panel with DAPI, a blue nuclear stain, heparan sulfate (green), and myc-B4GALT7 (red). Bar is $50 \mu \mathrm{m}$ for all panels

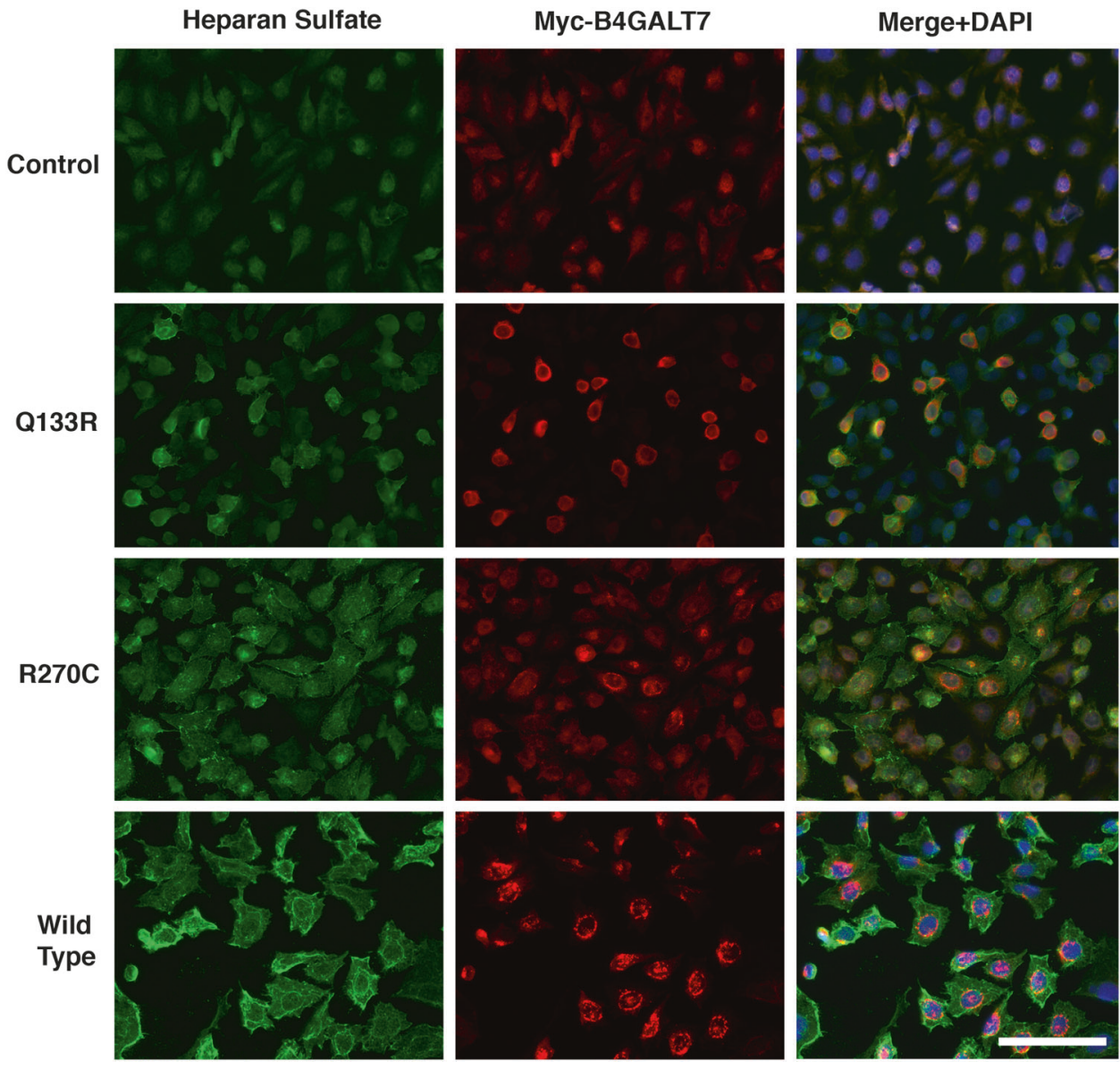


largely Golgi-like punctate pattern of intracellular staining for the B4GALT7-WT protein (Fig. 4). It is important to note in this regard that some proteoglycans are secreted proteins and so may be secreted and bind to nonB4GALT7-WT-expressing pgsB-618 cells in this context. B4GALT7-R270C-transfected pgsB-618 cells showed weaker myc staining than did B4GALT7-WT-transfected cells, but this mutant protein still largely appeared to be confined to a Golgi-like staining pattern. Similarly, heparan sulfate staining in B4GALT7-R270C-expressing cells was present, but in lower amounts than was seen for WT. Anti-myc staining of B4GALT7-Q133R-expressing pgsB-618 cells showed high protein expression, but with altered intracellular localization compared to WT or B4GALT7-R270C, and heparan sulfate staining was barely detectable above background levels. To confirm the altered subcellular localization, we repeated myc staining of transfected B4GALT7 variants with costaining for calnexin, a marker for the endoplasmic reticulum (Fig. 5), or with costaining for GM130, a marker for the Golgi apparatus (Fig. 6). Both B4GALT7-WT and B4GALT7R270C showed high colocalization with GM130 within expressing cells, while B4GALT7-Q133R showed high colocalization with calnexin.
We next made membrane preps from control CHO-K1 cells, pgsB-618 cells, and pgsB-618 cells stably overexpressing B4GALT7-WT, B4GALT7-R270C, or B4GALT7$Q 133 R$ and assayed them for B4GALT7 enzyme activity (Fig. 7). Quantitative western blots vs. known amounts of purified B4GALT7 protein were also done to normalize enzyme activity to the total amount of B4GALT7 protein present. We used p-nitrophenyl-xylopyranoside and UDP- $\left[1-{ }^{14} \mathrm{C}\right]$ galactose as substrates for this assay, and we separated reaction product from labeled substrate with SepPak C18 cartridges, largely as previously described $[11,12]$. Washes were also collected and counted to ensure that unused substrate was removed prior to elution of the reaction product. As expected based on previous reports [13], the known B4GALT7-R270C mutant showed a significant $(p<0.01)$ reduction in specific activity relative to B4GALT7-WT, but enzyme activity was still present, albeit reduced to $17 \pm 3 \%$ of WT activity and to $30 \pm 4 \%$ of activity in normal $\mathrm{CHO}$ cells. Specific activity of B4GALT7-WT in pgsB-618 cells was $1.8 \pm 0.2$ times that in WT CHO-K1 cells, while untransfected pgsB-618 cells had no detectable activity. The B4GALT7-Q133R mutant protein, in contrast to B4GALT7-WT and B4GALT7R270C, had little to no detectable enzyme activity above
Fig. 5 Localization of myc(B4GALT7) variants with calnexin, a marker for the endoplasmic reticulum. Immunostaining with anticalnexin (green) and anti-myctag antibody (red) in untransfected pgsB-618 cells and pgsB-618 cells stably transfected with B4GALT7-WT, B4GALT7-Q133R, or B4GALT7-R270C. Merge + DAPI shows a three-color panel with DAPI, a blue nuclear stain, calnexin (green), and mycB4GALT7 (red). Bar is $31 \mu \mathrm{m}$ for all panels

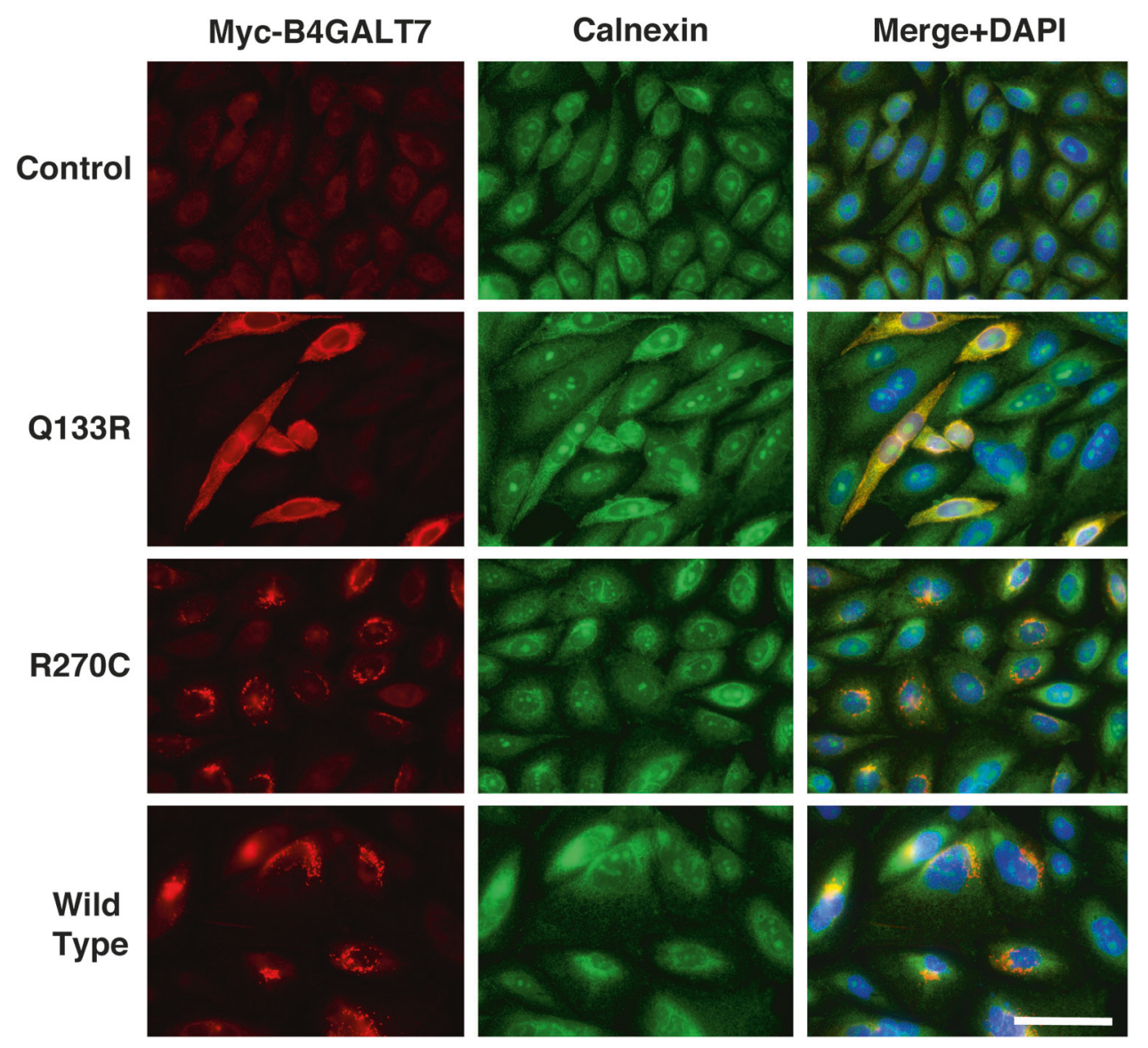


Fig. 6 Localization of myc(B4GALT7) variants with GM130, a marker for the Golgi apparatus. Immunostaining with anti-GM130 (green) and antimyc-tag antibody (red) in untransfected pgsB-618 cells and pgsB-618 cells stably transfected with B4GALT7-WT, B4GALT7-Q133R, or B4GALT7-R270C. Merge + DAPI shows a three-color panel with DAPI, a blue nuclear stain, GM130 (green) and mycB4GALT7 (red). Bar is $31 \mu \mathrm{m}$ for all panels

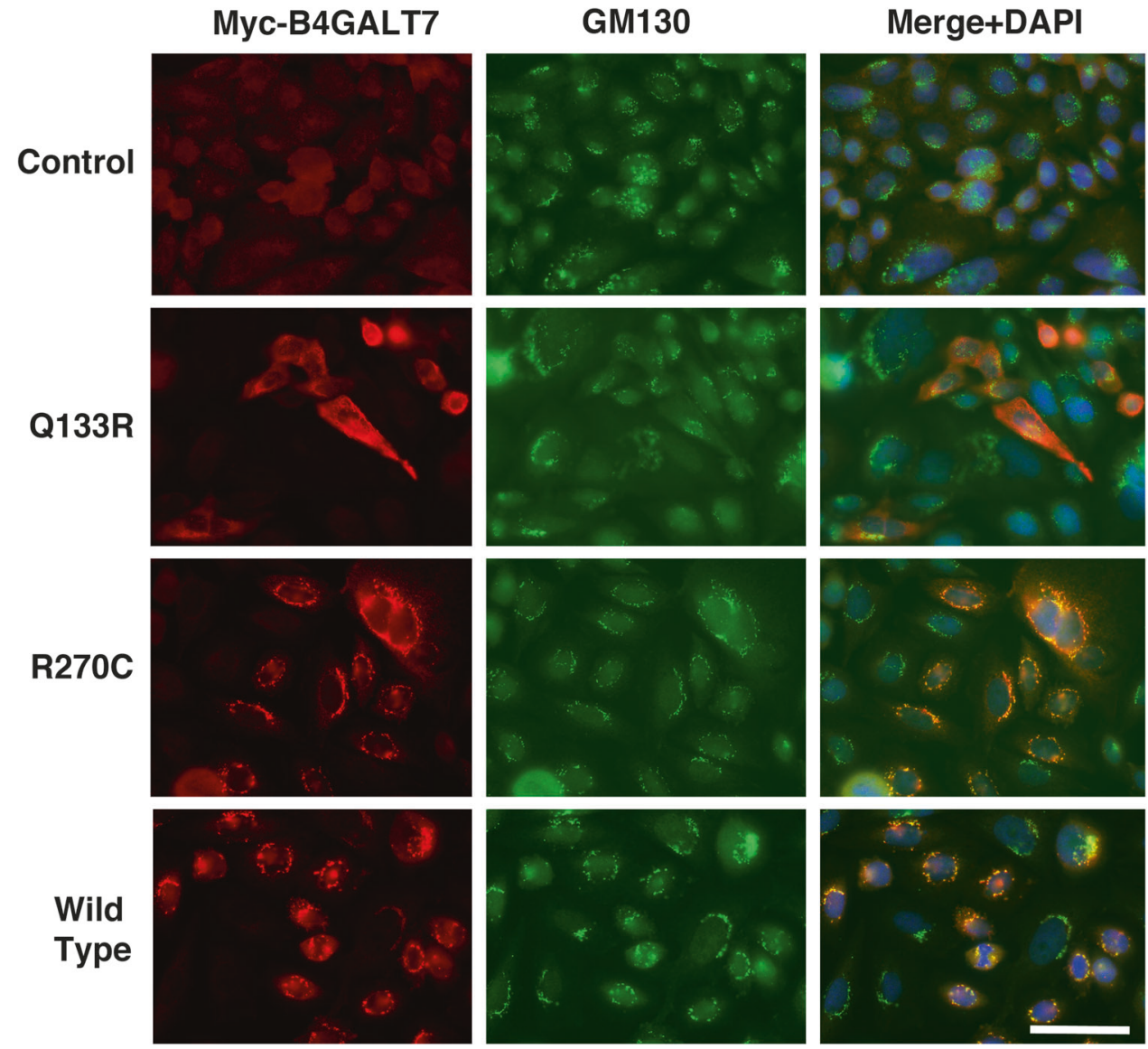

baseline levels $(0.8 \pm 0.3 \%$ of WT and $2 \pm 1 \%$ of normal CHO cells) (Fig. 7). As such, the p.(Q133R) variant appears effectively to be a null mutation within our ability to do these measurements.

\section{Discussion}

Linkeropathy is an umbrella term for conditions resulting in proteoglycan deficiency that are caused by defects in the synthesis of the common tetrasaccharide linker region, GlcA $\beta-1,3-$ Gal- $\beta 1,3-$ Gal- $\beta 1,4-X y l-\beta 1,3-$, that connects GAG chains to serines on the protein core $[1,3]$. Several enzymes are required for the synthesis of this common linker region, including xylosyltransferases 1 and 2 (XYLT1 and XYLT2), which can both contribute to the first xylose glycan, galactosyltransferase 1 (B4GALT7) and galactosyltransferase 2 (B3GALT6), which make the subsequent two galactose glycans (respectively), and glucuronosyltransferase 1 (B3GAT3), which makes the fourth glycan in the linker. B4GALT7 variants are exceedingly rare, and therefore, not well described [3, 4]. Many different terms have been used to describe individuals with pathogenic variants in this gene, including progeroid-type
Ehlers-Danlos syndrome (EDS, progeroid-type), EDS with short stature and limb anomalies, LRS, and the most recent classification of Spondylodysplastic Ehlers-Danlos syndrome (spEDS) [4, 14]. Recently, for certain B4GALT7related linkeropathies, such as spEDS, minimum criteria for diagnosis have been suggested, such as short stature and muscular hypotonia in the case of spEDS. However, spEDS can also be caused by variants in other genes (such as B3GALT6 and SLC39A13), and patients with LRS, with more of a skeletal dysplasia phenotype, are not considered to have an EDS phenotype but still fall into this category. Other features also overlap among patients with B4GALT7related disease, including radio-ulnar synostosis, low bone density, congenital contractures and dislocations, joint laxity, digital abnormalities, brachycephaly, small mouth, prominent eyes, short or webbed neck, congenital heart defects, and developmental delay [1,2]. Recent literature has suggested that all patients with a B4GALT7-related linkeropathy represent a spectrum of the same condition rather than distinct disease entities [4].

Our findings demonstrate a new phenotype associated with compound heterozygous pathogenic variants in B4GALT7 that include a novel variant p.(Q133R) and the previously described p.(R270C). While the proband 


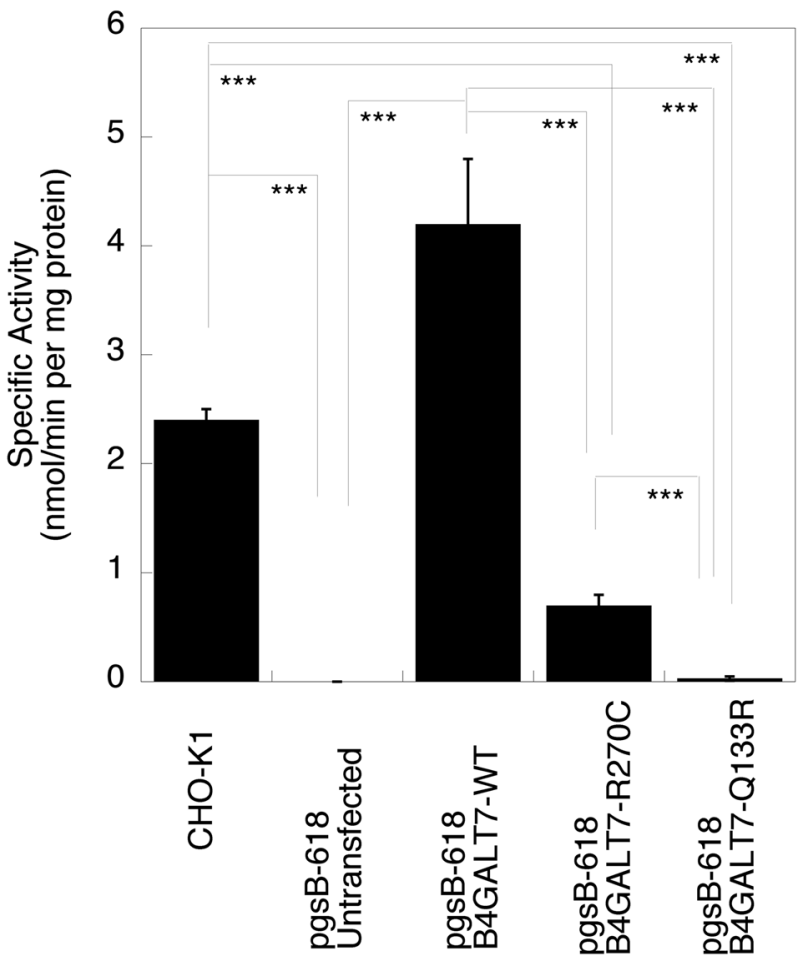

Fig. 7 Activity of B4GALT7 in stably transfected pgsB-618 cells. Untransfected normal CHO cells (CHO-K1), untransfected pgsB-618 cells, and pgsB-618 cells stably transfected with B4GALT7-WT, B4GALT7-R270C, and B4GALT7-Q133R were assayed for galactosyltransferase enzyme activity using p-nitrophenyl-xylopyranoside and UDP- $\left[1-{ }^{14} \mathrm{C}\right] \mathrm{Gal}$ as substrates. Errors are SEM for 3-6 replicates per group. $* * * p<0.001$

described in this paper has many of the features described in spEDS, such as short stature, short limbs, radio-ulnar synostosis, congenital contractures and dislocations, abnormal digits, as well as features of LRS (short limbs, bowed extremities), the skeletal features are much more severe than what has been previously described in any of these B4GALT7-related linkeropathies (Fig. 1). Specifically, the phenotype in this family is a prenatal or perinatal-lethal phenotype in three consecutive pregnancies.

Homozygous or compound heterozygous B4GALT7 variants associated with disease have not been previously described to cause a lethal phenotype. Seven diseasecausing variants have been reported previously, including one of the variants described in this paper p.(R270C) [5]. The individuals with the most severe phenotypes have had variants with demonstrated null function (p.(Leu206Pro)) or are expected to have absent function due to a frameshift duplication in conjunction with a moderate impairment in function (p.(Ala286Asp) and p.(R270C), respectively) $[5,13]$. While both individuals had severe short stature, skeletal dysplasia, osteopenia with fractures, joint hypermobility, abnormal healing, and cognitive delays, both were alive at the time of their case report at ages 5 and 3 years.

Functional studies on the novel p.(Q133R) variant presented here suggest that it is a null allele. Although the $\mathrm{p}$. Q133R protein was expressed at high levels in our cell-based assay system, 1.3 times that found in normal $\mathrm{CHO}$ cells, we found essentially no B4GALT7 enzyme activity or heparan sulfate expression for this protein. In addition, p.Q133R protein was colocalized with calnexin, a marker for the endoplasmic reticulum, rather than with GM130, a marker for the Golgi apparatus. This altered intracellular pattern differed from that of both B4GALT7 WT and p.R270C protein, which were colocalized with the Golgi marker despite being overexpressed by even greater amounts. The p.R270C variant showed reduced B4GALT7 activity, $17 \%$ of WT, but this was clearly greater than the nearly absent enzyme activity for p.Q133R. This level of reduction for p.R270C is greater than the reduction found in several previous reports, where p.R270C was 68\% [15] or 37\% [16] of WT enzyme activity. Greater reductions, however, to $20 \%$ or less of WT activity, were sometimes observed when p.R270C was assayed at cooler temperatures [16]. Regardless, our results support the notion that the severe loss of function of the p.(Q133R) variant, inherited as a compound heterozygote with the milder p.(R270C) variant, would have a more severe clinical phenotype than previously reported patients. Additional secondary impacts, separate from loss of enzyme function, may also result from the altered intracellular localization of the p.Q133R mutant protein.

The findings in this family expand the clinical phenotype of B4GALT7-related linkeropathies to include perinatal lethal skeletal dysplasia. Furthermore, radial ray defects (such as absent thumbs) and cleft palate, both of which are seen in this family, are not previously reported features of B4GALT7-related linkeropathies, and thus may also be considered an expansion of the clinical phenotypes associated with pathogenic variants in B4GALT7. This case highlights the utility of exome sequencing for individuals with skeletal dysplasia when typical skeletal dysplasia gene panel testing fails to identify the underlying cause.

Acknowledgements We thank this family for participation in research. Special thanks to Haley Guggenheim for technical support.

Funding This work was funded by the Abigail Wexner Research Institute at Nationwide Children's Hospital and supported by NIH grants R01 AR049722 and P50 AR070604 to PTM.

\section{Compliance with ethical standards}

Conflict of interest The authors declare that they have no conflict of interest. 
Publisher's note: Springer Nature remains neutral with regard to jurisdictional claims in published maps and institutional affiliations.

\section{References}

1. Jones KL, Schwarze U, Adam MP, Byers PH, Mefford HC. A homozygous B3GAT3 mutation causes a severe syndrome with multiple fractures, expanding the phenotype of linkeropathy syndromes. Am J Med Genet A. 2015;167A:2691-6.

2. Guo MH, Stoler J, Lui J, Nilsson O, Bianchi D, Hirschhorn JN, et al. Redefining the progeroid form of Ehlers-Danlos syndrome: report of the fourth patient with B4GALT7 deficiency and review of the literature. Am J Med Genet A. 2013;161A:2519-27.

3. Taylan F, Makitie O. Abnormal proteoglycan synthesis due to gene defects causes skeletal diseases with overlapping phenotypes. Horm Metab Res. 2016;48:745-54.

4. Ritelli M, Dordoni C, Cinquina V, Venturini M, Calzavara-Pinton P, Colombi M. Expanding the clinical and mutational spectrum of B4GALT7-spondylodysplastic Ehlers-Danlos syndrome. Orphanet J Rare Dis. 2017;12:153.

5. Salter CG, Davies JH, Moon RJ, Fairhurst J, Bunyan D, Study DDD, et al. Further defining the phenotypic spectrum of B4GALT7 mutations. Am J Med Genet A. 2016;170:1556-63.

6. Cartault F, Munier P, Jacquemont ML, Vellayoudom J, Doray B, Payet $\mathrm{C}$, et al. Expanding the clinical spectrum of B4GALT7 deficiency: homozygous p.R270C mutation with founder effect causes Larsen of Reunion Island syndrome. Eur J Hum Genet. 2015;23:49-53.

7. Arunrut T, Sabbadini M, Jain M, Machol K, Scaglia F, Slavotinek A. Corneal clouding, cataract, and colobomas with a novel missense mutation in B4GALT7-a review of eye anomalies in the linkeropathy syndromes. Am J Med Genet A. 2016;170:2711-8.

8. Kelly BJ, Fitch JR, Hu Y, Corsmeier DJ, Zhong H, Wetzel AN, et al. Churchill: an ultra-fast, deterministic, highly scalable and balanced parallelization strategy for the discovery of human genetic variation in clinical and population-scale genomics. Genome Biol. 2015;16:6.

9. Esko JD, Weinke JL, Taylor WH, Ekborg G, Roden L, Anantharamaiah G, et al. Inhibition of chondroitin and heparan sulfate biosynthesis in Chinese hamster ovary cell mutants defective in galactosyltransferase I. J Biol Chem. 1987;262:12189-95.

10. Boyle MP, Enke RA, Reynolds JB, Mogayzel PJ Jr., Guggino WB, Zeitlin PL. Membrane-associated heparan sulfate is not required for rAAV-2 infection of human respiratory epithelia. Virol J. 2006;3:29.

11. Lugemwa FN, Sarkar AK, Esko JD. Unusual beta-D-xylosides that prime glycosaminoglycans in animal cells. J Biol Chem. 1996;271:19159-65.

12. Okajima T, Fukumoto S, Furukawa K, Urano T. Molecular basis for the progeroid variant of Ehlers-Danlos syndrome. Identification and characterization of two mutations in galactosyltransferase I gene. J Biol Chem. 1999;274:28841-4.

13. Bui C, Talhaoui I, Chabel M, Mulliert G, Coughtrie MW, Ouzzine $\mathrm{M}$, et al. Molecular characterization ofbeta1,4-galactosyltransferase 7 genetic mutations linked to the progeroid form of Ehlers-Danlos syndrome (EDS). FEBS Lett. 2010;584:3962-8.

14. Malfait F, Francomano C, Byers P, Belmont J, Berglund B, Black J, et al. The 2017 international classification of the Ehlers-Danlos syndromes. Am J Med Genet C Semin Med Genet. 2017; 175:8-26.

15. Rahuel-Clermont S, Daligault F, Piet MH, Gulberti S, Netter P, Branlant $\mathrm{G}$, et al. Biochemical and thermodynamic characterization of mutatedbeta1,4-galactosyltransferase 7 involved in the progeroid form of the Ehlers-Danlos syndrome. Biochem $\mathrm{J}$. 2010;432:303-11.

16. Seidler DG, Faiyaz-Ul-Haque M, Hansen U, Yip GW, Zaidi SH, Teebi AS, et al. Defective glycosylation of decorin and biglycan, altered collagen structure, and abnormal phenotype of the skin fibroblasts of an Ehlers-Danlos syndrome patient carrying the novel Arg270Cys substitution in galactosyltransferase I (beta4GalT-7). J Mol Med. 2006;84:583-94. 